\title{
Modelos de responsabilidad social corporativa en el sector deporte. Una revisión sistemática \\ Models of corporate social responsibility in the sports sector. A systematic Review \\ Ingrid Fonseca, Jayson Bernate, Cristian Pérez \\ Corporación Universitaria Minuto de Dios (Colombia)
}

\begin{abstract}
Resumen. Las organizaciones deportivas sirven como plataforma para llevar a cabo transformaciones a nivel social, medio ambiental y económico y para promover el bienestar de las personas. De esta manera, se suscitan acciones sostenibles y en búsqueda del éxito organizacional se implementan mecanismos de regulación y se respeta la opinión de los grupos de interés. El objetivo de esta revisión sistemática es identificar y describir los modelos de responsabilidad social corporativa implementados en el sector deporte. Siguiendo la estructura del protocolo PRISMA se realizó una revisión sistemática en cuatro bases de datos (SportDiscus, Scopus, Scielo y Proquest), utilizando las palabras clave «corporate social responsibility», «model», «sport», «measuring» y «sport organization», el período de búsqueda fue de 10 años (2011-2020) y se seleccionaron un total de 15 artículos. Entre los resultados se encontró que los modelo de RSC en el sector deporte son escasos y los existentes se basan en escalas, cuestionarios y guiones de entrevista semiestructurada para conocer la opinión de los fanáticos, los deportistas y los gerentes. La evidencia científica indica la necesidad de implementar de manera efectiva los modelos de RSC porque son un canal de motivación, confianza y legitimidad con los deportistas, aficionados y grupos de interés, mejoran la imagen corporativa y la relación con la comunidad. Esta investigación presenta una línea de estudio para investigaciones futuras y sugerencias para que las organizaciones deportivas generen beneficios en los grupos de interés y promuevan valores.
\end{abstract}

Palabras Claves: modelo de gestión, modelo de evaluación, responsabilidad social, medición en el deporte, sostenibilidad.

\begin{abstract}
Sports organizations serve as a platform to carry out transformations at a social, environmental and economic level and to promote the well-being of people. In this way, sustainable actions are raised and in search of organizational success, regulatory mechanisms are implemented and the opinion of stakeholders is respected.The objective of this systematic review is to identify and describe the corporate social responsibility models implemented in the sports sector. Following the structure of the PRISMA protocol, a systematic review was carried out in four databases (SportDiscus, Scopus, Scielo and Proquest), using the keywords «corporate social responsibility», «model», «sport», «measuring» and «sport organization «, the search period was 10 years (2011-2020) and a total of 15 articles were selected. Among the results, it was found that CSR models in the sports sector are scarce and the existing ones are based on scales, questionnaires and semi-structured interview scripts to find out the opinion of fans, athletes and managers. Scientific evidence indicates the need to effectively implement CSR models because they are a channel of motivation, trust and legitimacy with athletes, fans and interest groups, improve the corporate image and the relationship with the community. This research presents a line of study for future research and suggestions for sports organizations to generate benefits for interest groups and promote values.
\end{abstract}

Keywords: management model, evaluation model, social responsibility, measurement in sport, sustainability.

\section{Introducción}

La responsabilidad social (RS) se relaciona con la importancia de generar valores con la finalidad de que la comunidad en general se involucre en la búsqueda de soluciones a problemáticas de la sociedad, es así como las personas son el punto de partida para la construcción de una comunidad (Vallaeys, 2006; Fonseca et al, 2020). Así mismo, se desarrolla para dar soluciones a las dificultades que se presentan a nivel económico, social y

Fecha recepción: 21-02-21. Fecha de aceptación: 03-10-21

Ingrid Fonseca

ingridfonsecaf@gmail.com ambiental, así como el respeto de los derechos y las normas legales, en pro del fortalecimiento de la economía, la sociedad y el medio ambiente, entre otros factores (Orozco et al., 2013). En este sentido, se deben fomentar cambios a nivel interior y exterior en las organizaciones para ser eficientes y sustentables, con la finalidad de dar respuesta a cada uno de los grupos de interés (Herrera, 2011).

Respecto a la responsabilidad social corporativa (RSC), la misma se formula con base en un grupo de estándares y lineamientos para responder a las necesidades de los trabajadores, sus familias y la comunidad, teniendo en cuenta aspectos laborales, sociales y del entorno, entre otros (Atehortua et al., 2008; Babiak, 
2010; Avendaño, 2013). En resumen, la RSC sirve para generar un crecimiento sustentable con base en la economía y el buen uso de los recursos y el entorno, siendo esta la encargada de guiar los procesos para dar soluciones a las necesidades que se presenten (Bradish \& Cronin, 2009; García, 2018). De igual forma, se caracteriza por ser un elemento indispensable para que las organizaciones contribuyan a la sociedad (Breitbarth \& Harris, 2008; Walker \& Parent, 2010).

Además, es una herramienta para mejorar la imagen corporativa, con la intención de promover un crecimiento positivo (Zhang \& Surujlal, 2015; Anderson \& Martin, 2019). Se espera que la RSC permee procesos relacionados con los derechos de las personas, el manejo frente a sobornos y el ambiente laboral teniendo en cuenta alianzas de empresas y la comunidad civil (Cortés, et al., 2012; Avendaño, 2013). En efecto, se deben generar soluciones en beneficio de los grupos de interés, mediante la prevención de problemas y la solución a efectos negativos que se puedan presentar a futuro (Bateman \& Snell, 2005).

\section{El deporte como herramienta de responsabili- dad social}

Por su parte, el deporte es una herramienta de innovación social que genera impactos a las comunidades (Montazeri et al., 2017; Mattera \& Baena, 2018; François et al., 2019), representa beneficios a las organizaciones (Mattera \& Baena, 2018) y tiene impactos positivos en los valores comunitarios, la integración cultural, la comunicación efectiva, la salud de las personas y la conciencia medio ambiental (Smith \& Westerbeek, 2007; Blumrodt et al., 2013; Breitbarth et al., 2015). Del mismo modo, en países en desarrollo guarda una estrecha relación con los objetivos sociales (Giulianotti, 2015) y coadyuda en la construcción de entornos amenos para deportistas y fanáticos (Devlin \& Sheehan, 2018; Schyvinck \& Willem, 2018).

Es así como, las organizaciones deportivas debido a su carácter social, sirven como plataforma de transformación en pro del desarrollo humano, generan aportes a la economía y al bienestar de las personas y llevan a cabo acciones filantrópicas y benéficas para fomentar la conciencia social (Babiak, 2010; Giulianotti, 2011; Heinze et al., 2014; Kim et al., 2019). Por otra parte, los equipos deportivos son un referente en procesos de planificación y enseñanza para las nuevas generaciones e involucran jugadores apasionados por lo que hacen (Babiak \& Wolfe, 2009).

Dichas características generan relaciones estrechas entre las organizaciones deportivas y los medios de comunicación, trabajadores, aficionados, comentaristas, patrocinadores, comités de organización, el Estado, entre otros (Babiak \& Wolfe, 2009; Sánchez-Sáez, 2018). Es decir, que los programas de RSC buscar una armonía, brindando mejores respuestas hacia los involucrados, en pro de una acertada toma de decisiones (Schiebel \& Pöchtrager, 2003, Heinze et al., 2014; Anderson \& Martin, 2019).

\section{La RSC y su relación con el deporte}

La responsabilidad social corporativa con relación al deporte adquiere mayor importancia, gracias a la intervención en la comunidad y la implementación de iniciativas que involucran la participación proactiva de las empresas deportivas como las visitas de jugadores profesionales a niños y niñas en centros de salud, la promoción de estilos de vida saludable y el fomento de programas de actividad física (Babiak \& Wolfe, 2009; Extejt \& Smith 2014; Breitbarth et al., 2015; Cobourn \& Frawley, 2017).

Breitbarth \& Harris (2008) consideran que deportes como el fútbol son primordiales para un desarrollo cultural, económico, social e incluyente y un espacio que cuenta con patrocinio, apoyo gubernamental y voluntariado. Es por ello, que los clubes profesionales se destacan a nivel global con relación a variables económicas y sociales, llevando al sostenimiento de las instituciones deportivas en el entorno empresarial (Breitbarth \& Harris, 2008; François et al., 2019; Moyo et al., 2020) y cada día los atletas, eventos y el turismo deportivo representan un instrumento de transformación social (Walzel et al., 2018; Leal Londoño, 2021).

Por su parte, López-Martínez \& Fernández-Fernández (2015) consideran necesario que los clubes de futbol como empresas que son, mantengan principios de transparencia, códigos de ética empresarial y den respuesta a sus obligaciones legales. Así mismo que realicen una revisión de su visión y misión, elaboren y publiquen informes de RSC y modifiquen la actitud pasiva que han tenido. En este sentido, se espera una buena praxis por parte de los dirigentes. De este modo, el llevar acciones de manera estratégica y relacional permite que las organizaciones obtengan ventajas al planificar, tengan un reconocimiento por parte de la sociedad y no actúen basados en la improvisación (Monserrat-Gauchi et al.,2020).

Para dar respuesta a dicha situación, Moyo et al., (2020) plantean la implementación de investigaciones para analizar los factores claves de la responsabilidad 
social corporativa, en especial en los países en desarrollo con la finalidad de darle el valor que se merece. A este respecto, se hace preponderante desarrollar modelos de RSC, de la misma forma que en sectores como el petrolero y el textil o el turismo deportivo porque tal y como lo plantea Alonso-Dos- Santos et al., (2017) cada uno tiene connotaciones y características específicas.

Dentro de ese marco, Montazeri et al., (2017), sostienen que al contar con dichos modelos se promueven acciones sostenibles, se tiene en cuenta la opinión del aficionado, se busca el éxito organizacional, se implementan acciones de regulación, se respeta y se tiene en cuenta la opinión de los grupos de interés.

Debido al auge de la sostenibilidad y la búsqueda de estrategias que integren los objetivos de desarrollo sostenible, desde la academia es necesaria una mayor producción científica que sustente las acciones a implementar para fortalecer el sector del deporte. En la actualidad, se resalta que los estudios sobre la responsabilidad social corporativa en el ámbito deportivo en Colombia y Latinoamérica son escasos. Hace falta generar una cultura al interior de las organizaciones para que estructuren su organigrama de tal manera que tengan programas o estrategias en pro de la RS. Por lo anteriormente expuesto, el objetivo de esta revisión sistemática es identificar y describir los modelos de responsabilidad social corporativa implementados en el sector deporte.

\section{Metodología}

En el proceso de obtención de información sobre los modelos de responsabilidad social corporativa implementados en el deporte, se realizó una revisión sistemática con base en la declaración PRISMA (Preferred Reporting Items for Systematic Reviews and Meta-Analyses) (Moher, et al, (2009). La búsqueda se realizó utilizando como palabras clave «corporate social responsibility», «model», «sport», «measuring», «sport organization» y como operador booleano AND. Para el proceso de selección de los documentos se establecieron los siguientes criterios de inclusión: 1) que sean redactados en idioma inglés; 2) que fueran artículos publicados en revistas científicas; 3) que estuvieran publicados en un límite de tiempo de 10 años (20112020). La revisión se llevó a cabo en las bases de datos Scopus, SportDiscus, Scielo y Proquest, estas se seleccionaron por ser repositorios internacionales de alto impacto a nivel académico y por tener herramientas de búsqueda avanzada. Se excluyeron artículos que no se ajustarán con el tema objeto de estudio y que estuvieran publicados en revistas sin comité de revisión, tesis, memorias y artículos de divulgación.

\section{Procedimiento}

Tal y como se observa en el diagrama de flujo en la figura 1, la población inicial fue de 175 artículos científicos y la muestra definitiva de 15 documentos. La selección se hizo siguiendo los lineamientos de la declaración PRISMA. En un primer momento se identificaron los artículos en las cuatro bases de datos seleccionadas, se eliminaron los documentos duplicados, se hizo una revisión de los títulos y resúmenes y para finalizar la lectura del texto completo de los artículos elegidos. La última revisión se llevó a cabo el 4 de noviembre de 2020 .

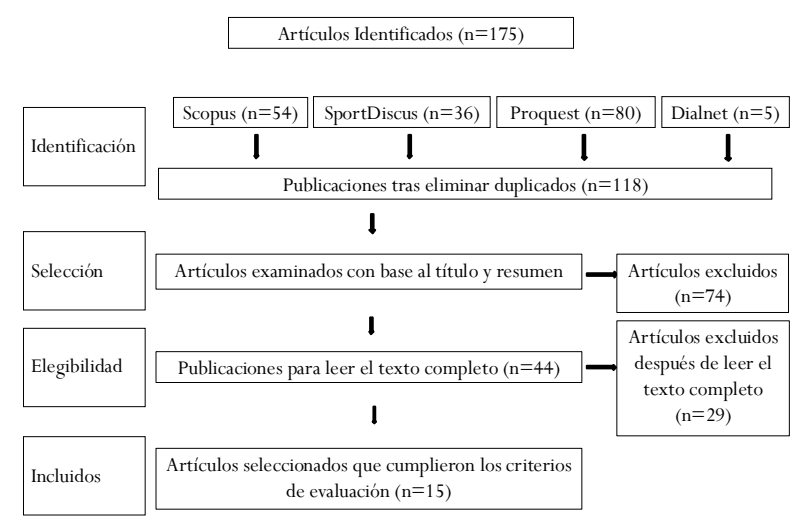

Figura 1. Proceso de selección de los artículos científicos.

\section{Resultados}

Mediante la búsqueda se identificaron 175 artículos, de los cuales se eliminaron 57 por estar duplicados, al revisar el título y el resumen se excluyeron 74 documentos, 44 fueron leídos de forma completa y 15 cumplieron los criterios de evaluación y constituyeron el corpus de estudio (Figura 1).

En la tabla 1 se pueden observar los resultados obtenidos, teniendo como criterio de análisis las referencias, las palabras clave, la muestra, el instrumento y las conclusiones de cada uno de los 15 estudios seleccionados.

\section{Discusión}

La presente revisión sistemática tuvo como objetivo identificar y describir los modelos de responsabilidad social corporativa implementados en el sector deporte. En este sentido, se resalta que la responsabilidad 
Tabla 1

\begin{tabular}{|c|c|c|c|c|}
\hline Autor / Año & Palabras Clave & Muestra & Instrumentos & Conclusiones \\
\hline $\begin{array}{l}\text { (Rayne, Leckie \& } \\
\text { McDonald, 2020) }\end{array}$ & $\begin{array}{l}\text { Asociaciones de RSC. } \\
\text { Comportamiento del consumidor. } \\
\text { Organizaciones sin fines de lucro. } \\
\text { Equipos deportivos profesionales. } \\
\text { Jerarquía de efecto. }\end{array}$ & $\begin{array}{l}504 \text { aficionados a equipos deportivos } \\
\text { y } 355 \text { patrocinadores de } \\
\text { organizaciones sin ánimo de lucro de } \\
\text { un equipo nacional de Cricket y una } \\
\text { organización sin ánimo de lucro para } \\
\text { víctimas de cáncer. }\end{array}$ & $\begin{array}{l}\text { Cuestionario con } 15 \text { dimensiones y } 43 \text { items, con } \\
\text { una escala Likert de } 1 \text { a } 7,(1=\text { muy en } \\
\text { desacuerdo / extremadamente improbable y } 7= \\
\text { muy de acuerdo / muy probable. }\end{array}$ & $\begin{array}{l}\text { La estimación del modelo estructural es adecuada, se tuvieron } \\
\text { en cuenta como dimensiones el conocimiento de la } \\
\text { organización, la identificación del usuario, las redes sociales, el } \\
\text { voz a voz, la intención de asistencia a los partidos, la compra de } \\
\text { mercancía, los juegos televisados y en vivo, la actitud y } \\
\text { sensibilización frente a la RSC. }\end{array}$ \\
\hline $\begin{array}{l}\text { (Lorgnier, Chanavat, Su } \\
\text { \& O'Rourke, 2020) }\end{array}$ & $\begin{array}{l}\text { Congruencia de valores. } \\
\text { Responsabilidad social corporativa. } \\
\text { Transferencia de valores. } \\
\text { Comité Olímpico Internacional. } \\
\text { Relación consumidor marca. }\end{array}$ & $\begin{array}{l}\text { Seis estudios a estudiantes } \\
\text { universitarios y familiares }(\mathrm{n}=1197) \\
\text { realizado en Estados Unidos y } \\
\text { Francia } \\
\text { antes y después de los Juegos } \\
\text { Olímpicos de } 2016 \text { y 2018. }\end{array}$ & $\begin{array}{l}\text { Cuestionario autoadministrado de } 6 \text { dimensiones } \\
\text { y } 24 \text { items, en una escala Likert de } 1 \text { a } 7(1=\text { no } \\
\text { importante y } 7 \text { =más importante }) \text {. }\end{array}$ & $\begin{array}{l}\text { Los hallazgos confirman la validez de la escala, así como su } \\
\text { confiabilidad y } \\
\text { consistencia interna en seis estudios, en diferentes contextos } \\
\text { culturales. } \\
\text { Los resultados sugieren que se puede utilizar una escala que } \\
\text { utilice los valores de una marca (o declaración de RSC) para } \\
\text { medir la congruencia entre la marca y el valor del consumidor. }\end{array}$ \\
\hline$(\mathrm{Yu}, 2020)$ & $\begin{array}{l}\text { Modelo de comportamiento dirigido al } \\
\text { cumplimiento de objetivos. } \\
\text { Teoría del comportamiento planificado. } \\
\text { Responsabilidad social corporativa. } \\
\text { Proceso de toma de decisiones. } \\
\text { Comportamiento del consumidor } \\
\text { deportivo. }\end{array}$ & $\begin{array}{l}\text { Se realizó una encuesta a } 408 \\
\text { espectadores que asistieron a juegos } \\
\text { de béisbol profesional celebrados en } \\
\text { Taiwán. } \\
\text { En total } 53,23 \% \text { eran hombres y } \\
46,77 \% \text { mujeres con una edad } \\
\text { media de } 26,32 \text { años. }\end{array}$ & $\begin{array}{l}\text { Cuestionario de } 10 \text { dimensiones y } 31 \text { ítems, en } \\
\text { una escala Likert de } 1 \text { a } 7,(1=\text { Totalmente de } \\
\text { acuerdo y } 7=\text { Totalmente en desacuerdo) }\end{array}$ & $\begin{array}{l}\text { Los hallazgos confirmaron que el modelo de medición se ajusta } \\
\text { a la información que se busca recolectar. Factores como la } \\
\text { actitud, la norma subjetiva, el control conductual percibido, la } \\
\text { emoción positiva anticipada, la frecuencia de la conducta pasada } \\
\text { desempeñaron papeles dominantes al influir para que las } \\
\text { personas decidan asistir a los juegos. }\end{array}$ \\
\hline $\begin{array}{l}\text { (McCullough, Orr \& } \\
\text { Watanabe, 2019) }\end{array}$ & $\begin{array}{l}\text { Impacto ambiental. } \\
\text { Comportamiento de los aficionados. } \\
\text { Evaluación del ciclo de vida. } \\
\text { Espectadores. } \\
\text { Organizaciones deportivas. }\end{array}$ & $\begin{array}{l}\text { Se analizan los informes de eventos } \\
\text { deportivos. }\end{array}$ & $\begin{array}{l}\text { Cuestionario de } 4 \text { dimensiones y } 29 \text { ítem teniendo } \\
\text { en cuenta los impactos directos y externos con } \\
\text { relación a la producción y el consumo. }\end{array}$ & $\begin{array}{l}\text { Este estudio identificó que en los eventos deportivos no se } \\
\text { o tienen en cuenta los aspectos ambientales, pero si se presta } \\
\text { atención a los impactos económicos. Se reconoce la necesidad } \\
\text { de ejercer mayor presión social para promover la sostenibilidad } \\
\text { dentro del deporte. }\end{array}$ \\
\hline $\begin{array}{l}\text { (Inoue, Funk \& } \\
\text { McDonald, 2017) }\end{array}$ & $\begin{array}{l}\text { Responsabilidad social corporativa } \\
\text { (RSC). } \\
\text { Intervención. } \\
\text { Compromiso. } \\
\text { Frecuencia de asistencia. } \\
\text { Deporte profesional. }\end{array}$ & $\begin{array}{l}634 \text { fanáticos de un equipo de fútbol } \\
\text { profesional australiano. } \\
75,6 \% \text { eran hombres y } 24,4 \% \text { eran } \\
\text { mujeres con edad media de } 46,5 \\
\text { años. }\end{array}$ & $\begin{array}{l}\text { Cuestionario de } 7 \text { dimensiones y un total de } 21 \\
\text { items en total, en una escala Likert de } 1 \text { a } 7,(1= \\
\text { Totalmente de acuerdo y } 7=\text { Totalmente en } \\
\text { desacuerdo) y evaluación de la asistencia de los } \\
\text { aficionados al estadio recopilados un año después. }\end{array}$ & $\begin{array}{l}\text { Se concluye que el constructo formulado es multifacético y } \\
\text { sirve para establecer la lealtad conductual y su relación con la } \\
\text { RSC. En este sentido, se debe entender como las acciones } \\
\text { responsables influyen en el comportamiento del cliente para } \\
\text { proporcionar una evaluación realista del retorno de la inversión } \\
\text { de las actividades de RSC. }\end{array}$ \\
\hline $\begin{array}{l}\text { (Montazeri, Talebpour, } \\
\text { Andam \& Kazemnejad, } \\
\text { 2017) }\end{array}$ & $\begin{array}{l}\text { Responsabilidad social. } \\
\text { Desarrollo sostenible. } \\
\text { Aficionados. } \\
\text { Grupos de interés. } \\
\text { Premier League. }\end{array}$ & $\begin{array}{l}640 \text { fanáticos en la Football Premier } \\
\text { League de fútbol de Irán. }\end{array}$ & $\begin{array}{l}\text { Cuestionario de } 5 \text { dimensiones y } 22 \text { ítems, en una } \\
\text { escala Likert de } 1 \text { a } 7,(1=\text { totalmente en } \\
\text { desacuerdo con la afirmación y } 7=\text { total acuerdo } \\
\text { con la declaración }) \text { y entrevistas a los aficionados. }\end{array}$ & $\begin{array}{l}\text { Los hallazgos sirven para incentivar a los equipos deportivos } \\
\text { para que fortalezcan los lazos con sus fanáticos para generar } \\
\text { aportes a las comunidades, realizando un apoyo a ONG's, } \\
\text { causas sociales, grupos vulnerables para mejorar la relación con } \\
\text { los grupos de interés. }\end{array}$ \\
\hline $\begin{array}{l}\text { Chang, Kang, Ko \& } \\
\text { Connaughton (2017) }\end{array}$ & $\begin{array}{l}\text { Orgullo. } \\
\text { Desempeño percibido del equipo por } \\
\text { parte de los fanáticos. } \\
\text { RSC. } \\
\text { Voz a voz. } \\
\text { Identificación del equipo. }\end{array}$ & $\begin{array}{l}540 \text { aficionados de la liga de béisbol } \\
\text { profesional de Corea del Sur. }\end{array}$ & $\begin{array}{l}\text { Cuestionario de } 5 \text { dimensiones (rendimiento del } \\
\text { equipo, RSC, orgullo, recomendación voz a voz e } \\
\text { identidad con el equipo) y } 15 \text { ítems. }\end{array}$ & $\begin{array}{l}\text { El estudio contribuye al conjunto de } \\
\text { conocimientos sobre las percepciones de los consumidores } \\
\text { deportivos sobre la RSC, la emoción (orgullo) y el } \\
\text { comportamiento (recomendación voz a voz). Se concluye que } \\
\text { el desempeño de los equipos dentro y fuera del campo mejora } \\
\text { el sentimiento de los fanáticos y la comunicación. }\end{array}$ \\
\hline $\begin{array}{l}\text { (Cobourn \& Frawley, } \\
2017 \text { ) }\end{array}$ & $\begin{array}{l}\text { Responsabilidad social de las empresas. } \\
\text { Deporte profesional. } \\
\text { Comunidad. } \\
\text { Gobernanza. }\end{array}$ & $\begin{array}{l}\text { Se recopilaron datos de } 22 \\
\text { ejecutivos y gerentes entrevistados } \\
\text { de } 12 \text { organizaciones deportivas } \\
\text { profesionales y sus departamentos } \\
\text { de RSC. }\end{array}$ & $\begin{array}{l}\text { Se llevaron a cabo entrevistas a los ejecutivos } \\
\text { sobre datos demográficos, planificación estratégica } \\
\text { y gobernanza. También se recopilación } \\
\text { documentos institucionales como misión y visión, } \\
\text { informes anuales, documentos de políticas, } \\
\text { boletines relacionados con las actividades o } \\
\text { estrategias } \\
\text { de RSC entre los años } 2012 \text { y } 2013 \text {. }\end{array}$ & $\begin{array}{l}\text { a Esta investigación proporciona conocimientos prácticos sobre } \\
\text { los } \\
\text { desafios y oportunidades en las organizaciones deportivas y } \\
\text { genera una perspectiva de gestión interna. } \\
\text { Se recomienda realizar estudiosz en diferentes contextos } \\
\text { deportivos internacionales y programas de RSC. }\end{array}$ \\
\hline $\begin{array}{l}\text { (Ch } \mid \text { ang, Ko, } \\
\text { Connaughton \& Kang, } \\
\text { 2016) }\end{array}$ & $\begin{array}{l}\text { Orgullo. } \\
\text { Apego. } \\
\text { RSC } \\
\text { Identificación del equipo. } \\
\text { Género. } \\
\end{array}$ & $\begin{array}{l}262 \text { aficionados de equipos } \\
\text { profesionales de béisbol y fútbol de } \\
\text { Corea del Sur. }\end{array}$ & $\begin{array}{l}\text { Cuestionario de } 4 \text { dimensiones (percepción de la } \\
\text { RSC, orgullo, identificación y apego al equipo) y } \\
14 \text { items. }\end{array}$ & $\begin{array}{l}\text { El estudio sirve para comprender la relación entre el apego y la } \\
\text { RSC. Se recomienda realizar estudios adicionales para } \\
\text { desarrollar una comprensión teórica del tema. }\end{array}$ \\
\hline (Sung \& Lee, 2016) & $\begin{array}{l}\text { Cáncer de mama. } \\
\text { Patrocinio. } \\
\text { Intención de compra. } \\
\text { Responsabilidad social corporativa } \\
\text { (RSC). } \\
\text { Imagen corporativa. } \\
\text { Evento deportivo. } \\
\end{array}$ & $\begin{array}{l}650 \text { mujeres con una edad media de } \\
41,2 \text { años, dentro del evento } \\
\text { deportivo benéfico, Maratón del } \\
\text { amor de la cinta rosada (PRLM) que } \\
\text { se llevó a cabo en Seúl, Corea del } \\
\text { Sur. }\end{array}$ & $\begin{array}{l}\text { Cuestionario de } 5 \text { dimensiones y } 16 \text { items en } \\
\text { total, en una escala Likert de } 1 \text { a } 7,(1=\text { Muy en } \\
\text { desacuerdo y } 7=\text { Muy de acuerdo })\end{array}$ & $\begin{array}{l}\text { Los hallazgos de esta investigación amplían el campo de acción, } \\
\text { al examinar las relaciones entre los patrocinadores y la } \\
\text { intención de compra en un entorno empresarial coreano. Se } \\
\text { resalta que la imagen corporativa se puede construir y } \\
\text { desarrollar a través de una estrategia de RSC efectiva que tenga } \\
\text { en cuenta cómo los participantes perciben la causa social y el } \\
\text { evento deportivo patrocinado. }\end{array}$ \\
\hline $\begin{array}{l}\text { (Huang ,Ye \& Kao, } \\
\text { 2015) }\end{array}$ & $\begin{array}{l}\text { Análisis de desempeño. } \\
\text { Responsabilidad social corporativa } \\
\text { (RSC). } \\
\text { Turismo deportivo. }\end{array}$ & $\begin{array}{l}133 \text { participantes de los eventos de } \\
\text { roadrunning organizados por la } \\
\text { Asociación China de Taipei Road } \\
\text { Running. } 77 \% \text { de género masculino } \\
\text { y edad media de } 35 \text { años. }\end{array}$ & $\begin{array}{l}\text { Cuestionario de tres partes, información } \\
\text { sociodemográfica, preguntas sobre la importancia } \\
\text { de la sostenibilidad de la RSC de las } \\
\text { organizaciones de acogida y el grado de } \\
\text { satisfacción de los participantes. Las dimensiones } \\
\text { fueron } 5 \text { y } 25 \text { items en total, con } 2 \text { escalas Likert } \\
\text { de } 1 \text { a } 5 \text {, una abordo los atributos de importancia } \\
\text { y la otra los atributos de desempeño. }\end{array}$ & $\begin{array}{l}\text { El estudio construye un marco de evaluación multidimensional } \\
\text { de los eventos de turismo deportivo con relación a la RSC, el } \\
\text { cual representa un método de valoración eficaz. Los resultados } \\
\text { indicaron que la organización anfitriona puede tener ventajas al } \\
\text { tadoptar la RSC y promover la preocupación social y el } \\
\text { desarrollo sostenible. }\end{array}$ \\
\hline $\begin{array}{l}\text { (Kihl, Babiak \& Tainsky, } \\
\text { 2014) }\end{array}$ & $\begin{array}{l}\text { Iniciativa de participación comunitaria } \\
\text { corporativa. } \\
\text { Responsabilidad social. } \\
\text { Deporte profesional. } \\
\text { Iniciativas sociales. } \\
\text { Estrategias. } \\
\text { Gestión de procesos. }\end{array}$ & $\begin{array}{l}\text { Se realizaron entrevistas a } 42 \text { partes } \\
\text { interesadas clave (ejecutivos de } \\
\text { equipo (3), socios corporativos (2), } \\
\text { entrenadores (6), jugadores (9) } \\
\text { padres de familia (10) y directores } \\
\text { de instalaciones deportivas (12) en } \\
\text { un equipo profesional de las } \\
\text { Grandes Ligas de Béisbol (MLB). } \\
\end{array}$ & $\begin{array}{l}\text { Se recopilaron datos primarios de las entrevistas } \\
\text { semiestructuradas y datos secundarios de } 150 \\
\text { documentos organizativos: comunicados de } \\
\text { prensa, páginas web y folletos. }\end{array}$ & $\begin{array}{l}\text { Se exploraron los procesos de implementación y gestión de una } \\
\text { iniciativa de apoyo a la comunidad por parte de un equipo } \\
\text { profesional. Se recomienda realizar investigaciones para } \\
\text { explorar el impacto de actividades de RSC por parte de las } \\
\text { organizaciones deportivas. }\end{array}$ \\
\hline $\begin{array}{l}\text { (Blumrodt, Dest } \\
\text { Bodin, 2013) }\end{array}$ & $\begin{array}{l}\text { Clubes de fútbol profesional. } \\
\text { RSC. } \\
\text { Imagen de marca. } \\
\text { Gestión estratégica de marca. } \\
\text { Entretenimiento deportivo. } \\
\text { Valor de marca basado en el cliente. } \\
\text { Fútbol. } \\
\text { Responsabilidad social. }\end{array}$ & $\begin{array}{l}500 \text { espectadores del club de fútbol } \\
\text { de Lorient y } 496 \text { espectadores del } \\
\text { club de Rennes, equipos franceses } \\
\text { de la Liga europea de fútbol } \\
\text { profesional. }\end{array}$ & $\begin{array}{l}\text { Entrevistas semi guiadas y cuestionario de } 4 \\
\text { dimensiones y } 30 \text { ítems, distribuido en tres } \\
\text { partes, la primera se centró en el } \\
\text { comportamiento de los espectadores (asistencia a } \\
\text { los partidos, abonado, VIP), la segunda estaba } \\
\text { vinculada a la medición de la imagen de marca y la } \\
\text { tercera al perfil del espectador (sexo, procedencia } \\
\text { y categoría socio profesional). }\end{array}$ & $\begin{array}{l}\text { Se deben implementar estrategias de RSC para comunicar los } \\
\text { esfuerzos a los grupos de interés y contribuir con el valor de } \\
\text { marca ya que los espectadores esperan un compromiso con la } \\
\text { sociedad y el entorno por parte de los clubes. Por otra parte, se } \\
\text { a propone realizar estudios sobre la convivencia en los estadios y } \\
\text { la calidad percibida. }\end{array}$ \\
\hline $\begin{array}{l}\text { (Breitbart, Hovemann } \\
\text { Walzel, 2011) }\end{array}$ & $\mathrm{NA}$ & $\begin{array}{l}\text { El estudio se basa en } 12 \text { entrevistas } \\
\text { semiestructuradas a directivos de } \\
\text { cinco clubes profesionales del futbol } \\
\text { europeo y sus principales } \\
\text { organizaciones socias de RSC. }\end{array}$ & $\begin{array}{l}\text { Se realizaron entrevistas semiestructuradas y se } \\
\text { analizaron sistemáticamente cuarenta y ocho sitios } \\
\text { web de clubes y se establecieron sugerencias } \\
\text { relevantes con relación a las perspectivas } \\
\text { económica, política integradora y ético } \\
\text { emocional teniendo en cuenta nueve indicadores } \\
\text { clave. }\end{array}$ & $\begin{array}{l}\text { El diseño del Cuadro de mando del desempeño de RSC busca } \\
\text { ayudar a los clubes de fútbol a desarrollar un enfoque } \\
\text { estratégico, lograr sus objetivos y controlar los impactos de sus } \\
\text { actividades a lo largo plazo. Se sugiere realizar más } \\
\text { investigaciones para adoptar e integrar acciones responsables } \\
\text { socialmente en organizaciones deportivas. }\end{array}$ \\
\hline (w & $\begin{array}{l}\text { Responsabilidad social. } \\
\text { Desarrollo de escala. } \\
\text { Prueba de modelos. } \\
\text { Actitudes del consumidor. }\end{array}$ & $\begin{array}{l}92 \text { espectadores de los Juegos All } \\
\text { Star de la liga nacional de Hockey } \\
\text { (NHL) y } 146 \text { de la Asociación } \\
\text { Nacional de carreras de automóviles } \\
\text { Stock Car (NASCAR) }\end{array}$ & $\begin{array}{l}\text { Cuestionario de } 3 \text { dimensiones y } 21 \text { ítems donde } \\
12 \text { se aplicaron a espectadores de la All Star de la } \\
\text { Liga Nacional de Hockey (NHL) y } 11 \text { de la } \\
\text { Asociación Nacional de Carreras de Automóviles } \\
\text { Stock Car (NASCAR) en Daytona Beach }\end{array}$ & $\begin{array}{l}\text { Los resultados de los dos estudios demostraron la influencia de } \\
\text { la emoción del fanático y el comportamiento de compra entre } \\
\text { los consumidores deportivos y se realizó para evaluar la } \\
\text { conciencia de RSC entre los consumidores. Se demostró que el } \\
\text { afecto hacia el equipo puede actuar como antecedente del } \\
\text { comportamiento real del consumidor. }\end{array}$ \\
\hline
\end{tabular}


social, día a día adquiere importancia y genera espacios de interés empresarial ya que se pueden evaluar los impactos de las organizaciones deportivas (Breitbarth et al., 2011).

Las investigaciones en este campo son realmente recientes y se espera contar con estudios que presenten modelos de RSC. Siendo así, Montazeri et al., (2017), plantean que resulta fundamental contar con escalas de medición. En este aspecto, se han realizado avances de manera progresiva, sin embargo, todavía queda bastante trabajo por realizar (Babiak, 2010).

Se ha podido constatar que existen pocos estudios que generen modelos para medir la responsabilidad social en el sector deporte y los instrumentos implementados son escalas, cuestionarios aplicados a fanáticos y guiones de entrevista semiestructurada dirigidos a grupos de interés y dirigentes deportivos (Montazeri et al., 2017; Yu, 2020; Kihl et al., 2014; Breitbarth et al., 2011; Sung \& Lee,2016).

Se resalta que, en los estudios realizados para medir la responsabilidad social corporativa, los cuestionarios y escalas de medición son las estrategias más implementadas. Por tanto, cumplen la función no solo de medir las actividades de la RSC sino también caracterizar las transformaciones que han tenido las marcas y las relaciones con las partes interesadas, entre otros (Lorgnier et al., 2020).

Los cuestionarios por su forma de creación o diseño se pueden ajustar a diferentes entornos y escenarios, para así garantizar una mejor comprensión (Lorgnier et al., 2020; Montazeri et al., 2017), con relación al mercado, los diferentes problemas a nivel social, ambiental y económico que se producen por las actividades realizadas y la implementación de estrategias por parte de las organizaciones deportivas, entre otros (Lorgnier et al., 2020; Chang et al., 2017; Kihl et al., 2014). De allí, surgen resultados eficaces que podrían ser estudiados, detallados y evaluados para mejorar los programas de RSC y así obtener beneficios a largo plazo en las estrategias y marketing de las organizaciones (Lorgnier et al., 2020). Es así como, se resalta que en la mayoría de investigaciones se toma en cuenta la opinión de los grupos de interés en pro del desarrollo sostenible, como forma eficaz para medir las actividades de RSC que se implementan (Montazeri et al., 2017).

Con base en los resultados obtenidos, a continuación, se presenta una clasificación de tres líneas sobre los artículos publicados: instrumentos para medir la RSC en el sector deporte, aportes de los modelos de la RSC en las organizaciones deportivas e implementación de la RSC.

\section{Instrumentos implementados para medir la RSC} en el sector deporte

Al hacer la revisión de los modelos se concuerda con lo planteado por Montazeri et al., (2017), quienes resaltan que se cuenta con variados instrumentos para evaluar la opinión y el comportamiento del aficionado frente a la organización teniendo en cuenta el impacto que se genera.

En su mayoría los modelos se diseñaron teniendo en cuenta la opinión del fanático (9) (Walker \& Heere, 2011; Blumrodt et al., 2013; Chang et al., 2016; Inoue et al., 2017; Montazeri et al., 2017; Chang et al., 2017; Rayne et al., 2020; Yu, 2020; Lorgnier et al., 2020), en todos estos estudios se implementaron cuestionarios para la recolección de la información con relación a la calidad percibida, el valor de la marca, la identidad con los equipos, la intención de compra, la percepción de los consumidores deportivos sobre las acciones de RSC que se llevan a cabo y la importancia de evaluar los impactos sociales, ambientales y económicos.

Por otra parte, dos estudios, que de igual forma tuvieron como instrumento el cuestionario, se basaron en la opinión de los participantes. En los mismos se resalta la necesidad de contar con un modelo de evaluación de eventos de turismo deportivo para conocer el nivel de satisfacción de los participantes y su opinión con relación a la sostenibilidad (Huang et al., 2015). Así como la relación entre los patrocinadores, la intención de compra, la imagen corporativa y el aporte que puede generar una estrategia de RSC efectiva (Sung \& Lee, 2016).

De igual forma, en dos estudios se tuvo en cuenta la percepción de los gerentes de clubes deportivos profesionales, en este caso se realizaron entrevistas semiestructuradas y se analizaron los sitios web y documentos institucionales. De esta manera, se obtuvieron datos prácticos sobre los desafíos en las organizaciones deportivas y se evidenció la importancia de contar con lineamientos de gestión interna para controlar los impactos a largo plazo (Breitbart et al., 2011; Cobourn \& Frawley, 2017). Entre tanto, Kihl et al., (2014) realizaron entrevistas a socios, directivos, entrenadores, jugadores, padres de familia y dirigentes de escenarios deportivos de un equipo profesional con la finalidad de evaluar el impacto de actividades de RSC por parte de las organizaciones deportivas y para finalizar McCullough et al., (2019) formularon un cuestionario para analizar los impactos directos e indirectos que se generan en los eventos deportivos. 
En este sentido, Lorgnier et al., (2020) presentan una escala que mide la relación que tienen las personas con el Comité Olímpico Internacional (COI). Por su parte, Montazeri et al., (2017) elaboraron un instrumento dirigido a seguidores del Football Premier League de Irán, donde tuvieron en cuenta variables económicas, éticas, ambientales, filantrópicas y legales, con la finalidad de medir la responsabilidad social corporativa, teniendo claro los ideales de la afición.

Walker \& Heere (2011), elaboraron una herramienta útil para medir los efectos de los esfuerzos sociales teniendo en cuenta el comportamiento del fanático y su fidelidad con el equipo. Huang et al., (2015), generaron un marco de evaluación multidimensional con base en atributos y dimensiones de evaluación relacionados con la RSC. Chang et al., (2017), buscaron examinar el impacto de la percepción de los fanáticos sobre el desempeño del equipo y la RSC teniendo en cuenta el orgullo que sienten y la recomendación voz a voz.

Por otra parte, Yu (2020) creó un modelo de medición basado en la intención del comportamiento, la emoción y la actitud de los fanáticos. De la misma manera, Rayne et al., (2020) formularon un modelo conceptual con base en la actuación del equipo, la opinión del aficionado, el patrocinador y la literatura sobre la responsabilidad social corporativa. Para finalizar Inoue et al., (2017) elaboraron un constructo teniendo en cuenta el comportamiento real, la lealtad conductual y la RSC percibida de los fanáticos de un equipo de fútbol profesional.

\section{Aportes de los modelos de la RSC en las organi- zaciones deportivas}

En la actualidad, la RSC representa un aspecto fundamental para el funcionamiento de las organizaciones deportivas y se espera que los gerentes estén a la vanguardia de la implementación de programas en pro de la comunidad (Montazeri et al., 2017). Por su parte autores como Rayne et al., (2020) y Bradish \& Cronin (2009) resaltan que las empresas del sector deporte se están asociando con organizaciones sin ánimo de lucro y ponen como ejemplo a Nike y su afiliación con Peace Players International, para implementar programas de inclusión.

Desde el punto de vista práctico, se espera que las ligas deportivas se centren en sus compromisos de responsabilidad social, teniendo como punto de partida la actividad física y el deporte por medio de acciones de voluntariado (Babiak, 2010). De esta forma, se buscan alianzas para solucionar las problemáticas y las necesi- dades de los grupos de interés (Babiak, 2010; Huang et al., 2015; Montazeri et al., 2017; Chang et al., 2017). Día a día aumentan acciones innovadoras por parte de los equipos profesionales, que se ven reflejados en aportes a la comunidad, los fanáticos, las distintas organizaciones sin ánimo de lucro, entre otros (Kihl et al., 2014; François et al., 2019).

Se resalta que la RSC sirve para mejorar la fidelidad del fanático y representa una nueva estrategia de marketing al generar lazos emocionales que influyen de manera positiva a las organizaciones deportivas (Inoue et al., 2017;Yu, 2020). Así mismo, la importancia en el logro del deporte, realizar acciones justas y el cambio constante con intenciones de mejorar, son clave en el proceso de posicionamiento de la organización (Hamil et al., 2010; Chang et al., 2016).

La mayoría de estudios establecen que la RSC ha tomado valor porque los gerentes dan soluciones a problemáticas reales, toman decisiones (Blumrodt et al., 2013; Huang et al., 2015; Montazeri et al., 2017) y se generan efectos positivos en el sector deporte e impactos económicos para dar respuesta a las necesidades de la sociedad, promoviendo el cuidado del entorno y finalmente la utilización de recursos reutilizables (Montazeri et al.,2017; Lorgnier et al., 2020). Siendo de esta manera, un factor primordial para el crecimiento de autoconfianza del aficionado y la promoción de conductas y emociones para fortalecer la fidelización (Bradish \& Cronin, 2009; Chang et al., 2017; Yu, 2020).

En este sentido, Chang et al., (2017) plantean que las acciones socialmente responsables repercuten en la identificación de los trabajadores con la empresa, es así como los jugadores pueden ser voluntarios en programas deportivos, y promover estilos de vida saludable en la comunidad. Así mismo, Chang et al., (2016), consideran que se crean emociones positivas, se genera apego con los equipos, se da una buena imagen e información positiva.

Entre tanto, Lorgnier et al., (2020), afirman que las marcas deportivas internacionales se basan en la promoción de valores para mejorar las relaciones entre el equipo y el consumidor y se formulan estrategias de RSC para fortalecer la lealtad, la satisfacción y el lazo afectivo. Babiak \& Kihl (2018), afirman que los equipos profesionales deben centrarse en iniciativas de RSC, al ser una plataforma para lograr la identificación de los fanáticos con los equipos y un medio de comunicación efectiva.

Para finalizar, Kihl et al., (2014) y Montazeri et al., (2017), consideran que los modelos de RSC sirven para 
que las organizaciones se involucren con las comunidades, se creen lazos de conexión con los jóvenes, gobiernos locales, organizaciones sin ánimo de lucro y fundaciones a través de actividades de índole social, educativo y filantrópico. Por ello, es importante reconocer la importancia de implementar estrategias que apunten a objetivos concretos, para regular las consecuencias de las acciones que se llevan a cabo teniendo en cuenta los impactos internos y externos (Breitbarth et al., 2011; Blumrodt, et al., 2013; Rayne et al., 2020). De esta forma se da respuesta a los grupos de interés y se tiene un mejor manejo a nivel económico, para generar beneficios internos y externos y mejores resultados deportivos (Montazeri et al.,2017; Chang et al., 2016).

\section{Implementación de la RSC en las organizacio- nes deportivas}

Es importante implementar modelos de responsabilidad social corporativa y observar las transformaciones que se pueden presentar para contar con grupos de trabajo preocupados por los impactos que producen (Kihl et al., 2014; Huang et al., 2015). La ejecución de dichos programas nace del empeño voluntario de los gerentes para dar un manejo adecuado al medio ambiente y la sociedad (Blumrodt et al., 2013; McCullough et al., 2019). En este sentido, se espera que las organizaciones deportivas tengan fundaciones o departamentos encargados de la RSC y generen iniciativas frente al manejo estructural de los clubes deportivos (Chang et al., 2016; Cobourn \& Frawley, 2017).

Es por ello que las organizaciones impactan a los grupos de interés generando aportes con relación a la imagen del equipo y la integración de actividades que reflejan el manejo correcto de la $\mathrm{RS}$, para incentivar espacios de promoción de valores y respeto de los derechos de las personas por parte de equipos profesionales o ligas deportivas (Babiak, 2010; Kihl et al., 2014; Rayne et al., 2020).

$\mathrm{Al}$ respecto Inoue et al., (2017), afirman que la RSC se relaciona con la lealtad conductual y actitudinal, por lo tanto, es necesario diseñar, comunicar y activar acciones para facilitar el bienestar de los fanáticos. En consecuencia, es primordial que los dirigentes de los equipos deportivos se enfoquen en la comunicación de las iniciativas desarrolladas a través de anuncios y promociones en los eventos deportivos. Acciones como donaciones, patrocinio y voluntariado deportivo brindan oportunidades únicas porque se involucra al público y los participantes, hay contribuciones sociales y se desarrolla apego hacia los equipos (Sung \& Lee, 2016;
Sánchez-Sáez, 2018).

Los estudios analizados demuestran que involucrarse con los fanáticos, los deportistas y los grupos de interés (Walker \& Heere, 2011) generar programas de RSC en eventos pequeños y brindar charlas de estilos de vida saludable permite conocer e impactar a las sociedades (Sánchez-Sáez, 2018; McCullough et al., 2019). Implementar herramientas de responsabilidad social permite crear procesos innovadores que dejen resultados comerciales, promuevan la participación en el mercado, aumenten las ganancias y fortalezcan la reputación de la marca (Hamil et al., 2010; Giulianotti, 2015; Cobourn \& Frawley, 2017).

Dentro de este marco, las empresas desean ser consideradas responsables con la sociedad e implementan programas para mejorar la visibilidad, es así como invierten en acciones transformadores en ámbitos de actividad física, deporte, educación, salud, cultura y la comunicación de las mismas con el apoyo de los grupos de interés internos y externos (Giulianotti, 2015; Sung \& Lee, 2016; McCullough et al., 2019, Bernate et al., 2019), de esta forma es necesario implementar lineamientos y declaraciones que estén en congruencia con los contextos específicos de cada organización para evidenciar su impacto en la sociedad (Walzel et al., 2018; Lorgnier et al., 2020).

El presente estudio presenta algunas limitaciones. La primera se relaciona con que la investigación se basó en modelos de responsabilidad social corporativa, en consecuencia, se recomienda realizar estudios futuros sobre el impacto de la RSC en el deporte profesional, programas comunitarios y eventos deportivos. La segunda fue que la revisión se hizo en idioma inglés y la última limitación se sustenta en la muestra, lo cual representa una dificultad al momento de hacer comparaciones, sin embargo, al ser un tema relativamente nuevo en Latinoamérica, los resultados sirven de antecedentes para investigaciones futuras.

\section{Conclusiones}

Como se puede observar, la formulación e implementación de modelos de RSC se hizo por medio de escalas, cuestionarios y guiones de entrevista semiestructurada dirigidos a los grupos de interés y los gerentes de las organizaciones deportivas. Este estudio ha identificado que los modelos traen beneficios para las organizaciones deportivas con relación a la imagen corporativa ya que la RSC es una plataforma para generar valor en la sociedad a nivel económico, social y cultural. 
De igual forma, es un canal de motivación, confianza y legitimidad con los trabajadores, se transmiten valores, se promueven estilos de vida saludable, se contribuye al desarrollo de la infraestructura deportiva y se mejora la relación con los fanáticos.

Los resultados de esta revisión sistemática permiten inferir que los modelos de RSC son una herramienta de marketing, dejan beneficios comerciales, mejoran la reputación de la marca y la lealtad por parte de los trabajadores y aficionados y contribuyen para entender el comportamiento de los grupos de interés y su reacción ante las problemáticas sociales, económicas y ambientales.

En resumen, en la literatura consultada se plantea la necesidad de que los dirigentes deportivos integren en sus prácticas empresariales estos instrumentos validados de medición de la RS para cumplir los objetivos y metas planteadas, dar respuesta a los impactos a largo plazo y realizar auditorías.

Basado en los hallazgos de la literatura científica, es conveniente señalar que, se requiere mayor comprensión de estos modelos de RSC con la finalidad de integrarlos en las organizaciones deportivas y darle una mayor importancia teniendo en cuenta el contexto, el tamaño de las organizaciones o eventos, la opinión de los patrocinadores, empleados, aficionados, el impacto de sus actividades y el retorno de la inversión en acciones de RS. Entre las implicaciones prácticas, se resalta que el implementar modelos de responsabilidad social corporativa en el sector deporte permite consolidar y fortalecer los procesos administrativos y la relación con los grupos de interés.

El tema de la RSC en el deporte es relativamente nuevo en el contexto latinoamericano, por lo que esta revisión sirve como antecedente para elaborar investigaciones puntuales.

Es conveniente concluir que se requieren más estudios al ser un tema de alto interés para la gestión del deporte y una línea de estudio que puede ser implementada para buscar nuevas alternativas de desarrollo empresarial en las organizaciones deportivas.

\section{Referencias}

Alonso-Dos-Santos, M., Calabuig, F., Montoro, F., \& Valantine, I. (2017). Influencia de la RSC en la transmisión de imagen en el patrocinio turístico deportivo. Revista Iberoamericana de Psicología del ejercicio y el deporte, 12(1), 23-33

Anderson S. \& Martin M. (2019). The African American Community and Professional Baseball: Examining Major
League Baseball's Corporate Social Responsibility Efforts as a Relationship Management Strategy. International Journal of Sport Communication, 12(3), 397-418. https:// doi.org/10.1123/ijsc.20180157

Atehortua F., Bustamante R. \& Valencia J. (2008). Modelos de Gestión. Sistema de gestión integral. Una sola gestión, un solo equipo, Editorial Universidad de Antioquia. Medellín, Colombia. Recuperado de: https://bit.ly/2GQPPgQ Avendaño, W. (2013) Responsabilidad social (RS) y responsabilidad social corporativa (RSC): una nueva perspectiva para las empresas. Revista lasallista de investigación, 10(1), 152. Recuperado de: https://bit.ly/3ePWDHZ

Babiak, K. (2010). The role and relevance of corporate social responsibility in sport: A view from the top. Journal of Management and Organization, 16(4), 528. https:// doi.org/10.5172/jmo.2010.16.4.528

Babiak, K., \& Kihl, L. A. (2018). A case study of stakeholder dialogue in professional sport: An example of CSR engagement. Business and society review, 123(1), 119-149. https://doi.org/10.1111/basr.12137

Babiak, K., \& Wolfe, R. (2009). Determinants of corporate social responsibility in professional sport: Internal and external factors. Journal of sport management, 23(6), 717742. https://doi.org/10.1123/jsm.23.6.717

Bateman T. y Snell S.(2005), Administración: una ventaja competitiva. McGrawHill/Interamericana (4), 150151 Recuperado de: https: / /bit.ly/2Io44dx

Bernate, J., Fonseca, I., \& Betancourt, M. (2019). Impacto de la actividad física y la práctica deportiva en el contexto social de la educación superior (Impact of physical activity and sports practice in the social context of higher education). Retos, 37, 742-747. https://doi.org/ 10.47197/retos.v37i37.67875

Blumrodt J., Desbordes M., \& Bodin D. (2013). Professional football clubs and corporate social responsibility. International Journal, 3(3), 205-225.https://doi.org/ 10.1108/sbm0420110050

Bradish, C., \& Cronin, J. J. (2009). Corporate social responsibility in sport. Journal of Sport Management, 23(6), 691-697.

Breitbarth T., \& Harris P. (2008). The role of corporate social responsibility in the football business: Towards the development of a conceptual model. European Sport Management Quarterly, 8(2), 179-206. https://doi.org/ 10.1080/16184740802024484

Breitbarth T., Heere B., Walker M., Kellison T., \& Heinze K. (2015). Corporate social responsibility in sport. North American Society for Sport Management Conference, 298-299. Recuperado de: https://bit.ly/3nd4fHF

BreitbarthT.,Walzel S., Anagnostopoulos C., \&Van F. (2015). Corporate social responsibility and governance in sport: 
«Oh, the things you can find, if you don't stay behind!». Corporate Governance:The International Journal of Business in Society, 15, 254-273. https://doi.org/10.1108/ cg0220150025

Breitbarth, T., Hovemann, G., \& Walzel, S. (2011). Scoring strategy goals: Measuring corporate social responsibility in professional European football. Thunderbird International Business Review, 53(6), 721737.https://doi.org/10.1108/ sd.2012.05628eaa.014

Chang M., Kang J., KoY., \& Connaughton D. (2017). The effects of perceived team performance and social responsibility on pride and wordofmouth recommendation. Sport Marketing Quarterly, 26(1), 31.Recuperado de: https: / /bit.ly/2IlpgB3

Chang M., KoY., Connaughton D., \& Kang J. (2016). The effects of perceived CSR, pride, team identification, and regional attachment: the moderating effect of gender. Journal of Sport\&Tourism, 20(2), 145-159.https: / / doi.org/ 10.1080/14775085.2016.1193822

Cobourn S., \& Frawley S. (2017). CSR in professional sport: An examination of community models. Managing Sport and Leisure, 22(2), 113-126. https: / / doi.org/10.1080/ 23750472.2017.1402694

Cortés Á., Romero G., Zabala N. (2012). Documento referencial para la implementación de un programa de responsabilidad social empresarial en cooperativas (Trabajo de grado de pregrado, Universidad Ean). Recuperado de: https://bit.ly/3eMtXiU

Devlin M., \& Sheehan K. (2018). A «Crucial Catch» examining responses to NFL teams' corporate social responsibility messaging on Facebook. Communication \& Sport, 6(4), 477-498. https://doi.org/10.1177/ 2167479517719683

Extejt M., \& Smith E. (2004). Leadership Development through Sports Team Participation, Journal of Leadership Education, 8 (2), 224-237. https:/ /doi.org/10.12806/ $\mathrm{v} 8 / \mathrm{i} 2 / \mathrm{rf} 7$

François, A., Bayle, E., \& Gond, J. P. (2019). A multilevel analysis of implicit and explicit CSR in French and UK professional sport. European Sport Management Quarterly, 19(1), 15-37.

Fonseca, I., Bernate, J., Betancourt, M., Barón, B., \& Cobo, J. (2019) Developing Social Responsibility in University Students. Proceedings of the 201911 th International Conference on Education Technology and Computers, 215-218.

García J. (2018). El Pacto Mundial, Punto de Convergencia en la Línea del Tiempo del Desarrollo Sostenible y la Responsabilidad Social Empresarial y su Influencia en las Empresas. Dictamen Libre, (23), 71-88. https://doi.org/ $10.18041 / 26194244 /$ dl. 23.5146
Giulianotti R. (2011). The sport, development and peace sector: A model of four social policy domains. Journal of social policy, 40(4), 757-776. https: / /doi.org/10.1017/ s0047279410000930

Giulianotti, R. (2015). Corporate social responsibility in sport: critical issues and future possibilities. Corporate Governance, 15(2), 243-248.https: / /doi.org/10.1108/ CG-10-2014-0120

Hamil, S., Walters, G., \& Watson, L. (2010). The model of governance at FC Barcelona: balancing member democracy, commercial strategy, corporate social responsibility and sporting performance. Soccer \& Society, 11(4), 475-504.

Heinze, K. L., Soderstrom, S., \& Zdroik, J. (2014). Toward strategic and authentic corporate social responsibility in professional sport: A case study of the Detroit Lions. Journal of Sport Management, 28(6), 672-686. https: / / doi.org/10.1123/jsm.20130307

Herrera H. (2011). Responsabilidad social y ética empresarial. Ecoe Ediciones, 658. Recuperado de: https://bit.ly/ 32vTW9u

Huang F., Ye Y., \& Kao C. (2015). Developing a novel Intuitionistic Fuzzy Importance-performance Analysis for evaluating corporate social responsibility in sport tourism event. Expert Systems with Applications, 42(19), 6530-6538. https://doi.org/10.1016/ j.eswa.2015.04.008

Inoue Y., Funk D., \& McDonald H. (2017). Predicting behavioral loyalty through corporate social responsibility: The mediating role of involvement and commitment. Journal of Business Research, 75, 46-56.https://doi.org/ 10.1016/j.jbusres.2017.02.005

Kihl, L., Babiak, K., \& Tainsky, S. (2014). Evaluating the implementation of a professional sport team's corporate community involvement initiative. Journal of Sport Management, 28(3), 324-337.https: / /doi.org/10.1123/ jsm.20120258

KimY., Magnusen M., Weaver A., \& Kim M. (2019). Rural community wellbeing through minor league sport. Sport, Business and Management, 9(4), 328-343.https: / /doi.org/ $10.1108 / \mathrm{sbm} 1020180092$

Leal-Londoño, M., Travé-Molero, R., Medina, F. X., AbadiaNaudí, S., \& Sánchez-Bergara, S. (2021). Turismo deportivo: Una oportunidad para los territorios locales en un contexto global. Un estudio Delphi (Sport tourism: an opportunity for local regions in a global context. A Delphi study). Retos, 42, 77-88. https://doi.org/ 10.47197/retos.v42i0.8669

López-Martínez, R., \& Fernández-Fernández, J. L. (2015). Responsabilidad Social Corporativa y Buen Gobierno 
en los clubes de fútbol españoles. Universia Business Review, (46), 38-53.

Lorgnier, N. G., Chanavat, N., Su, C. J., \& O’Rourke, S. M. (2020). Examining the influence of brandbased value congruity: do the values of the International Olympic Committee really matter? Service Business, 14(1), 73 99.https: / /doi.org/10.1007/s11628019004091

Mattera M., \& Baena V. (2018). The Ball is in Your Court: Using Socially Responsible Actions as an Effective Marketing Tool. In Sports Media, Marketing, and Management: Breakthroughs in Research and Practice, 140-154. https: / / doi.org/10.4018/9781522554752.ch008

McCullough, B. P., Orr, M., \& Watanabe, N. M. (2019). Measuring externalities: The imperative next step to sustainability assessment in sport. Journal of Sport Management, 35(5), 393-402.https://doi.org/10.1123/ jsm. 20190254

Moher, D., Liberati, A., Tetzlaff, J., Altman, D., \& Prisma Group. (2009). Preferred reporting items for systematic reviews and metaanalyses: the PRISMA statement. PLoS medicine, 6(7), e1000097. doi:10.1186/ 2046405341

Monserrat-Gauchi, J., González-Redondo, P., \& CampilloAlhama, C. (2020). La comunicación organizacional en las entidades deportivas náuticas: Propuesta de modelo estratégico y relacional. Retos: nuevas tendencias en educación fisica, deporte y recreación, (38), 692-699.

Montazeri A., Talebpour M., Andam R., Kazemnejad A. (2017). Measuring corporate social responsibility in sport industry: Development and validation of measurement scale. Annals of Applied Sport Science, 5 (2), 97-114. https: / /doi.org/10.18869/acadpub.aassjournal.5.2.97

Moyo, T., Duffett, R., \& Knott, B. (2020). Environmental Factors and Stakeholders Influence on Professional Sport Organizations Engagement in Sustainable Corporate Social Responsibility: A South African Perspective. Sustainability, 12 (11), 4504. https: / / doi.org/10.3390/ su12114504

Organización Internacional del Trabajo OIT. (2007). Declaración Tripartita de Principios Sobre las Empresas Multinacionales y la Política Social. 3a. International Labour Organization, Recuperado de: https: / / bit.ly/2ImPJya

OrozcoY., Acevedo M., \& Acevedo J. (2013). Responsabilidad Social Empresarial: Teorías, índices, estándares y certificaciones. Cuadernos de administración, 29 (50), 196206. https: / / doi.org/10.25100/cdea.v29i50.55

Rayne D., Leckie C., \& McDonald H. (2020). Productive partnerships? Driving consumer awareness to action in CSR partnerships. Journal of Business Research, 118, 49 57. https://doi.org/10.1016/j.jbusres.2020.06.037
Sánchez-Sáez, J.A., Segado-Segado, D., \& Vidal, A. (2018). Sports events socially responsible as the engine for local development. Journal of Sports Economics \& Management 8(3), 172-186.

Schiebel W., \& Pöchtrager S. (2003). Corporate ethics as a factor for successthe measurement instrument of the University of Agricultural Sciences (BOKU), Vienna. Supply Chain Management: An International Journal, 8(2), 116-121.https://doi.org/10.1108/13598540310468715 Schyvinck C., \& Willem A. (2018). A typology of causerelated marketing approaches in European professional basketball. Sport Management Review, 21(4), 347-362.https://doi.org/10.1016/j.smr.2017.08.006

Smith A., \& Westerbeek H. (2007). Sport as a vehicle for deploying corporate social responsibility. Journal of corporate citizenship, (25), 43-54. https://doi.org/ 10.9774/gleaf.4700.2007.sp.00007

Sung M., \& Lee W. (2016). What makes an effective CSR program? An analysis of the constructs of a causerelated participant sport sponsorship event. International Journal of Sports Marketing and Sponsorship, 17 (1), 56-77. https: / /doi.org/10.1108/ijsms022016004

Vallaeys F. (2006). Breve marco teórico de responsabilidad social universitaria., Responsabilidad Social Universitaria. Recuperado de: https: / /bit.ly/36oQOgO

Viveros J., \& Maigual J. (2012). Modelo de responsabilidad social para la empresa privada con ánimo de lucro. Tendencias, 13(1), 116-134. Recuperado de: https: / /bit.ly/ 3n8oar6

Walker M., \& Heere B. (2011). Consumer attitudes toward responsible entities in sport(CARES): Scale development and model testing. Sport Management Review, 14(2), 153166.https: / /doi.org/10.1016/j.smr.2010.08.001

Walker M., \& Parent M. (2010). Toward an integrated framework of corporate social responsibility, responsiveness, and citizenship in sport. Sport Management Review, 13(3), 198213. https://doi.org/10.1016/ j.smr.2010.03.003

Walzel, S., Robertson, J., \& Anagnostopoulos, C. (2018). Corporate social responsibility in professional team sports organizations: An integrative review. Journal of Sport Management, 32(6), 511-530.

Yu C. (2020). The role of CSR in sport consumption decisionmaking. Marketing Intelligence \& Planning. https: / /doi.org/10.1108/mip0220200060

Zhang, Z., \& Surujlal, J. (2015). Willingness of sport fans to participate in socially responsible community programmes of professional sport organisations. South African journal for research in sport, physical education and recreation, 37(3), 185-197. Recuperado de: https:/ /bit.ly/ 36t9RH4 